\title{
Measurement of Neutrino's Magnetic Monopole Charge, Vacuum Energy and Cause of Quantum Mechanical Uncertainty
}

Eue Jin Jeong ( $\nabla$ ejeong1@gmail.com )

Tachyonics Research Institute

Dennis Edmondson

Columbia College, Marysville, Washington

\section{Research Article}

Keywords: fundamental physics, elementary particle physics, electricity and magnetism, experimental physics

Posted Date: October 9th, 2020

DOI: https://doi.org/10.21203/rs.3.rs-88897/v1

License: (c) (i) This work is licensed under a Creative Commons Attribution 4.0 International License.

Read Full License 


\title{
Measurement of Neutrino's Magnetic Monopole Charge, Vacuum Energy and Cause of Quantum Mechanical Uncertainty
}

\begin{abstract}
Charge conservation in the theory of elementary particle physics is one of the bestestablished principles in physics. As such, if there are magnetic monopoles in the universe, the magnetic charge will most likely be a conserved quantity like electric charges. If neutrinos are magnetic monopoles, as physicists have speculated the possibility, then neutrons must also have a magnetic monopole charge, and the Earth should show signs of having a magnetic monopole charge on a macroscopic scale. To test this hypothesis, experiments were performed to detect the magnetic monopole's effect near the equator by measuring the Earth's radial magnetic force using two balanced high strength neodymium rods magnets that successfully identified the magnetic monopole charge. From this observation, we conclude that at least the electron neutrino which is a byproduct of weak decay of the neutron must be magnetic monopole. We present mathematical expressions for the vacuum electric field based on the findings and discuss various physical consequences related to the symmetry in Maxwell's equations, the origin of quantum mechanical uncertainty, the medium for electromagnetic wave propagation in space, and the logistic distribution of the massive number of magnetic monopoles in the universe. We elaborate on how these seemingly unrelated mysteries in physics are intimately intertwined together around magnetic monopoles.
\end{abstract}

\section{Introduction}

In 1988, J.J. Steyaert published a conference paper titled "The Neutrino as a Tachyonic Non-charged Light Magnetic Monopole?" [1]. Alan Chodos et al discussed in their paper "The neutrino as a tachyon" in 1985 [2] and E. Recami on the same issues in 1978 [3]. Much earlier, O.M.P. Bilaniuk, V.K. Deshpande and E.C.G. Sudarshan already discussed the possibility of the existence of tachyon in 1962 [5].

Dirac theorized early on that for electric charge to be quantized, a magnetic monopole must exist [6]. Many years later, in 1974, Polyakov [7] and 't Hooft [8] discovered that the existence of monopoles follows from quite general ideas about the unification of fundamental interactions. Some GUTs [9], such as the Pati-Salam model [10] and superstring theory [11], predict the existence of magnetic monopoles as well. Despite the ongoing measurements of the neutrino's mass, it becomes increasingly evasive, suggesting the possibility that neutrinos may be tachyons. Recently, in 2019, the joint experimental team of UK, South Africa, Spain, France, and Brazil published 
reporting that the lightest neutrinos have an upper bound mass of $0.086 \mathrm{eV}$ with a $95 \%$ confidence level [12].

Undoubtedly, the questions of whether neutrinos are tachyons, whether there are magnetic monopoles in the universe, or whether neutrinos are both tachyons and magnetic monopoles have been an enduring mystery in physics for quite some time.

In this paper, we present new experimental evidence that neutrinos are light magnetic monopoles by measuring the Earth's magnetic monopole charge based on the general charge conservation principle in particle physics, the results of which may prove automatically that neutrinos are also tachyons.

In conventional nuclear beta decay processes, a neutron decays into a proton, electron, and an anti-neutrino as follows.

$$
{ }_{0}^{1} n \rightarrow{ }_{1}^{1} p+{ }_{-1}^{0} e+{ }_{0}^{0} \bar{v}_{e}
$$

The electric charge and baryon number are conserved in the process, while the neutrino carries the left-over energy and momentum.

The unanswered question is "Could it be possible that the individual neutrons have magnetic monopole charge of small quantity if a neutrino is a magnetic monopole?"

\section{Experimental Principle}

Neutrons exist in substantial numbers in the atomic elements on Earth in their composite nuclear structures. In essence, the task of measuring the magnetic monopole charge of neutrino becomes the task of measuring the Earth's magnetic monopole charge based on the assumption that magnetic charge is conserved. If a perfectly balanced high strength

long cylindrical test magnet is placed in the horizontal position on the surface of the earth having pivot at its center, the Earth's magnetic monopole will make the assembly tilt toward one side or the other with the magnitude of tilting force being dependent on the strength of the Earth's magnetic monopole charge.

On the other hand, the Earth's geomagnetic field, as shown in images [14] [15], runs horizontal to the surface of the Earth with a vertical component in both the northern and southern hemispheres that can obscure the data when measuring only the Earth's magnetic monopole effect. Therefore, the first task is to find the location in the world where the vertical component of the dipole geomagnetic field is negligible.

We accomplished this through a novel sensor design that uses two different cylindrical neodymium magnet sets having different diameters and lengths by stacking neodymium disk magnets. After carefully weighing each of the halves of the long dipole magnet assembly and balancing the weight of each separately on a precision micro scale, the center of the dipole magnet was placed on the pivot of tight string made of nonmagnetic 
material with negligible thickness to ensure minimum torque resistance on the magnet rod assembly to minimize obscure experimental data.

If the Earth does not have any magnetic monopole charge, the well-balanced neodymium magnet rod should remain balanced in the horizontal position. Any tilting force on the sensor magnets can be measured in the horizontal position using the precision micro scale to reflect the collective magnetic monopole charge of the neutrons on Earth.

\section{Measurement and Analysis of Experimental Data}

We decided to choose Cuenca Ecuador a city close to the equator with high altitude. In October 2018, we traveled to Cuenca equipped with two sets of long neodymium cylindrical magnets, a precision magnetometer (WT10A), a precision digital micro scale (AMOTGR 2001) and a well-thought-out balancer. The coordinate location where the measurement was made is $(-2.899350,-78.989264)$ at Avenue Gonzalez Suarez and Calle Jacinto in Cuenca Ecuador.

One of the assembled neodymium magnets has a length of $19 \mathrm{~cm}$ and a diameter of 10 $\mathrm{mm}$, where both sides have magnetic fields of the same magnitude measured by the sensitive Gauss meter (WT10A), and the other neodymium dipole magnet has a length of $16.6 \mathrm{~cm}$ and a diameter of $12 \mathrm{~mm}$. The earth as a monopole magnet will exert magnetic force on these test magnets by pulling down on one side and pushing up the other when the balanced dipole magnet is placed horizontally and resting at the center on a tight string of negligible thickness.

If consistent tilting occurs on the balance test of two different monopole sensors, it indicates that the earth has indeed magnetic monopole charge stemming from the accumulative monopole effect of its neutrons.
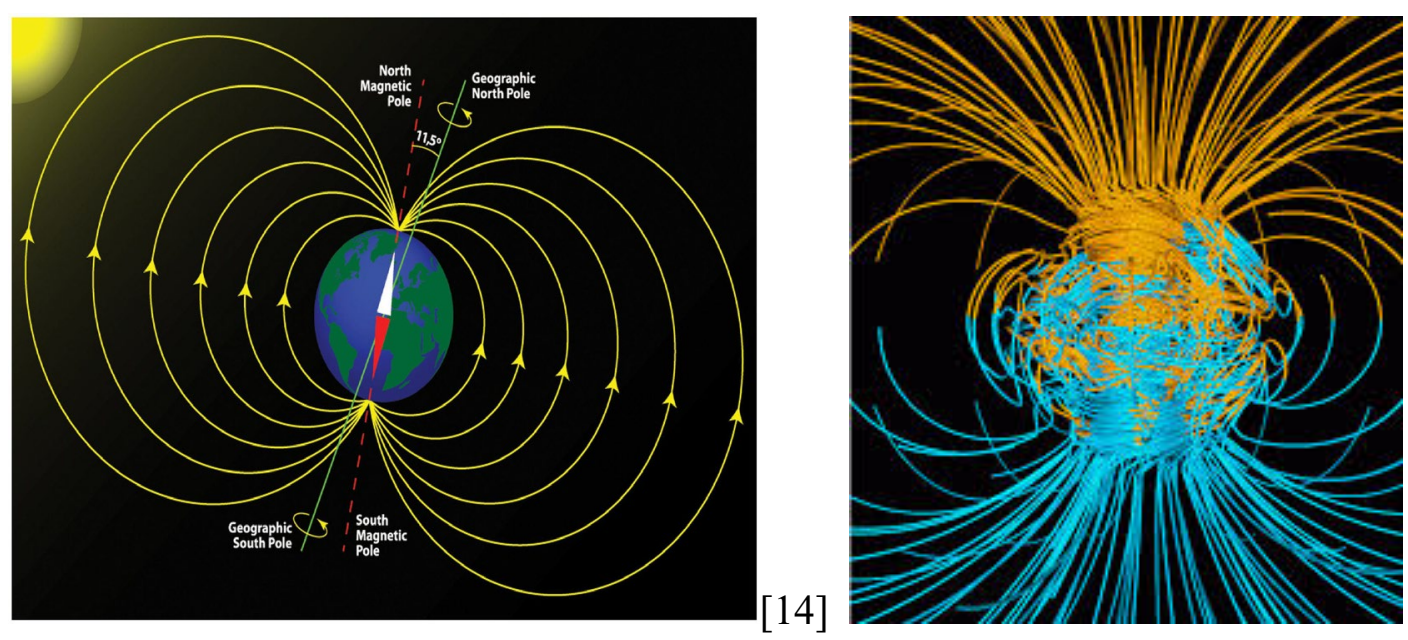
The above pictures of the geomagnetic field lines show that in the northern and southern hemispheres, the vertical component of the magnetic field is present because the geomagnetic field line is not strictly horizontal to the surface of the Earth in those regions. Image [15] depicts computer simulated Earth's magnetic dipole in the hypothetical case of pole reversal, which is significant because the prominent vertical component of the Earth's magnetic field can be populated in large areas of the Earth.

The basic equation of the force between two different permanent magnet poles $q_{m 1}$ and $q_{m 2}$ is

$$
F=\frac{\mu_{0} q_{m 1} q_{m 2}}{4 \pi r^{2}}
$$

which is identical in form to the equation for the force between two different electrostatic charges [15].

To determine the magnetic strength of the test magnets, $F_{1}$ is measured at the moment when each half of the full length of the test magnets is pulled to be separated at the preset separation gap $r_{1}$. The separation distances $r_{1} 5.2 \mathrm{~mm}$ and $5.1 \mathrm{~mm}$ are chosen for no specific reason other than to make sure the gap distance is not too close or too far so that the measured results do not obscure the actual strength of the test magnets. $1.2 \mathrm{~kg}$ and $0.65 \mathrm{Kg}$ of weight equivalent horizontal magnetic pulling forces are measured (5)

$$
F_{1}=\frac{\mu_{0} q_{m} q_{m}}{4 \pi r_{1}^{2}}
$$

for the magnetic charge strength of the two test magnets. It turns out that using this method to measure the strength of each magnetic pole is more direct and reliable than using the result of magnetic flux density measured by a Gauss meter and converts it into the magnetic charge strength.

The next step is to measure how much strength the magnetic monopole of the earth has and then calculate the estimated number of neutrons in the entire earth, and we will be able to obtain the single magnetic monopole charge of the individual neutron and subsequently that of the individual neutrino.

If we write the magnetic monopole charge of the Earth $Q_{m}$, the tilting force due to the interaction between the Earth magnetic monopole and the dipole magnet placed on the horizontal pivot at the center is given by

$$
F_{2}=\frac{2 \mu_{0} q_{m} Q_{m}}{4 \pi R^{2}}
$$

The factor of 2 comes from the two sides, one from the attractive force between N-S and the other from the repulsive force between $\mathrm{N}-\mathrm{N}$ on the opposite side of the test magnet. 
The elevation at the test site of Cuenca Ecuador is $2.56 \mathrm{~km}$ above sea level. Hence, $R=(6368+2.56) \mathrm{Km}$ and the downward tilting weights measured at the horizontal position of the magnets are $0.78 \mathrm{~g}$ and $0.52 \mathrm{~g}$, respectively, on the digital micro scale for the two test magnets (5).

\section{Measurement Data}

$\begin{array}{llllllll}\text { Rod Magnet } & \text { length } & \text { diameter } & F_{1} & r_{1} & F_{2} & B_{0} & \text { Weight R/L } \\ \text { Rod Magnet } 1 & 16.6 \mathrm{~cm} & 12 \mathrm{~mm} & 1.2 \mathrm{~kg} & 5.2 \mathrm{~mm} & 0.78 \mathrm{~g} & 454 \mathrm{mT} & 71.81 \mathrm{~g} / 71.84 \mathrm{~g} \\ \text { Rod Magnet } 2 & 19 \mathrm{~cm} & 10 \mathrm{~mm} & 0.65 \mathrm{~kg} & 5.1 \mathrm{~mm} & 0.52 \mathrm{~g} & 413 \mathrm{mT} & 56.83 \mathrm{~g} / 56.86 \mathrm{~g}\end{array}$

The south pole side of the magnet tilts downward consistently for both test Rod Magnet1 and Rod Magnet2, indicating that the Earth is a north magnetic monopole. "Weight R/L" is the weight of half of each test magnet separately measured before assembling the two halves into the full length. The weight of each half of the test magnets is measured in the vertical position with the $\mathrm{S}$ pole side down on the micro scale placed on top of the threeinch-tall nonmagnetic foam to minimize the magnetic interference on the electronic micro scale.

In both cases, the difference in weight of the right and left sides of the rod magnet remained within $0.03 \mathrm{~g}$ after balancing, and this weight difference is considered spread evenly along the length of the magnets. We found negligible differences in the magnitude of the measured $F_{2}$ after flipping both halves of the test rod magnets from $\mathrm{N}$ to $\mathrm{S}$ and vice versa.

\section{Analysis}

Using the data obtained after repeated measurements, we found $Q_{m}=2.75 \times 10^{16} \mathrm{Weber}$ for the Earth's monopole magnetic charge of the north kind measured by Rod Magnet1 and $Q_{m}=2.54 \times 10^{16}$ Weber for the same north kind measured by Rod Magnet2. We chose the average of the two and determined $Q_{m}=2.645 \times 10^{16}$ Weber for the north magnetic monopole charge of the Earth.

To estimate the total number of neutrons on Earth to calculate the individual magnetic monopole charge of the neutron, we used the element table published by the CRC handbook of Chemistry and Physics 97th edition [16].

For 99.9 percent of the mass on Earth according to the element table, 


$\begin{array}{llcc}\% & \text { Element } & \text { \# Neutron/Proton } & \text { Weighted Average N/P } \\ & & & \\ 5.63 & \text { Iron } & 30 / 26 & 168.9 / 146.38 \\ 46.1 & \text { Oxygen } & 8 / 8 & 368.8 / 368.8 \\ 28.2 & \text { Silicon } & 14 / 14 & 394.8 / 394.8 \\ 2.33 & \text { Magnesium } & 12 / 12 & 27.96 / 27.96 \\ 8.23 & \text { Aluminum } & 14 / 13 & 115.22 / 106.99 \\ 4.15 & \text { Calcium } & 20 / 20 & 83 / 83 \\ 2.36 & \text { Sodium } & 12 / 11 & 28.32 / 25.96 \\ 2.09 & \text { Potassium } & 20 / 19 & 41.8 / 39.71 \\ 0.565 & \text { Titanium } & 26 / 22 & 14.69 / 12.43 \\ 0.095 & \text { Manganese } & 30 / 25 & 2.85 / 2.375 \\ 0.14 & \text { Hydrogen } & 0 / 1 & 0 / 0.14\end{array}$

The mass difference due to the isotopes is considered in each element's average atomic mass.

We find the percentage distribution of the mass of the Earth to be $50.7 \%$ neutron and $49.3 \%$ proton, and the contribution from electrons is negligible. Using the known total mass of the Earth $5.972 \times 10^{24} \mathrm{Kg}$ and the mass of a single neutron $1.675 \times 10^{-27} \mathrm{Kg}$, we find the best estimated number of neutrons on the Earth $\# n_{\text {earth }}=1.8076 \times 10^{51}$.

Hence, the single magnetic monopole charge $m_{v}$ of a neutron is calculated to be

$$
m_{v}=\frac{Q_{m}}{\# n_{\text {earth }}}=1.463 \times 10^{-35} \text { Weber } .
$$

Further investigation illustrated that the repulsive monopole magnetic force between two matter objects is $1.14 \times 10^{-13}$ times smaller than the attractive gravity force between them, assuming that the same number of neutrons comprised each of the two matter objects. This indicates that the repulsive magnetic monopole force among the stars and planets is negligible compared to that of gravity on a galactic scale.

The noted discrepancy of this finding from the results of past studies is that the measured magnetic monopole charge of the neutron does not match Dirac's prediction of $g=\frac{N}{2} \frac{\hbar c}{e}$ [19], where $h$ is Planck's constant and $N$ is an integer resulting in the calculated Dirac's magnetic monopole charge $N \times 0.99 \times 10^{-7}$.

However, if Dirac's magnetic monopole is taken for the magnetic monopole charge of a neutron, the repulsive magnetic force between matter objects becomes too large to be ignored in celestial mechanics. Newtonian mechanical description of planetary motion will not work in such cases. 
There is a difference in the order of $10^{28}$ between Dirac's and the present report of the magnetic monopole charge $1.463 \times 10^{-35}$ Weber . Dirac's magnetic monopole looks rather close to the value $g=N \frac{\mu_{0}}{4 \pi}$, where $\mu_{0}=4 \pi \times 10^{-7} \mathrm{H} / \mathrm{m}$ is vacuum permeability.

This brings us to the relation $h=\frac{\mu_{0} e}{c}$ and by assuming $\mu_{0}=n_{0} m_{v}$ where $m_{v}$ the magnetic monopole charge of the neutrino and $n_{0}$ is the vacuum magnetic monopole flux number density of the neutrinos in a given volume, we have recovered the form $m_{v}=\frac{1}{n_{0}} \frac{h c}{e}$ for magnetic monopole where $n_{0}=8.589 \times 10^{28}$.

\section{Neutron Beta Decay Process Including Magnetic Monopoles}

Assuming that the conservation of magnetic charge is fully valid in the universe, the neutron beta decay can now be written as

$$
{ }_{0}^{1} n_{1} \rightarrow{ }_{1}^{1} p_{0}+{ }_{-1}^{0} e_{0}+{ }_{0}^{0} \overline{\boldsymbol{v}}_{e 1}
$$

where the low right-hand side sub-indices indicate the number of conserved magnetic monopole charges. The $\mathrm{W}$ boson is identified as a temporary composite transient particle that has both magnetic and electric charges before splitting into an electron and antineutrino. As J.J. Steyaert pointed out in his paper [1], the weak interaction in the standard model could be a manifestation of the magnetic monopole effect in the nuclear interaction processes.

\section{Estimated Speed of Non-Stationary Magnetic Monopoles in the Universe}

To estimate the speed of the background neutrinos, we assume that the electric field created by the moving tachyonic magnetic monopole neutrinos must be strong enough to counteract the attractive electrostatic force from the proton inside the atomic hydrogen.

The reasoning for this assumption is that there are no known physical principles other than electromagnetism that can account for the mechanical stability of the atomic structure in vacuum because the fundamental interaction between the electron and proton in various atoms is still electromagnetic in nature apart from the quantum mechanical uncertainty.

The electric field created by the traveling magnetic monopole may be written as 


$$
\vec{E}=\frac{1}{4 \pi \varepsilon_{0}} \frac{m_{v} \vec{v} \times \hat{r}}{r^{2}}
$$

where $m_{v}$ is the magnetic monopole charge and $\vec{v}$ is its velocity.

The electric field (10) created by a moving magnetic monopole is presently not known because an isolated magnetic monopole has not been identified. However, if a traveling magnetic monopole does exist in the form of a neutrino (9), the electric field it creates is expected to have the same form as the known magnetic field

$$
\vec{B}=\frac{\mu_{0}}{4 \pi} \frac{q \vec{v} \times \hat{r}}{r^{2}}
$$

created by moving electric charge $q$ with speed $\vec{v}$, if we assume the full symmetry of Maxwell's equation between electricity and magnetism.

The tacit assumption in equation (10) is that Maxwell's equation permits the speed of travel for magnetic monopole particles without restriction.

The speed of matter particles has encountered limit $c$ by the introduction of special relativity later by Einstein. However, Maxwell's equations are not subject to this restriction because there is no known first principle that limits the speed of charged particles in Maxwell's theory of electricity and magnetism.

If the vacuum electric field (10) created by traveling magnetic monopoles can affect the motion of the electron that is about to collapse into the proton of the hydrogen atom, it must have an equivalent field strength as the attractive electrostatic field from the proton

$$
E=\frac{1}{4 \pi \varepsilon_{0}} \frac{e}{r^{2}}
$$

The two electric fields (10) and (12) are geometrically different because one (10) is cylindrical and the other (12) is spherical. However, depending on the frequency of many directionally varying magnetic monopoles passing by the proton, the two fields can counteract each other and keep the electron in a constant agitating and rotating mode.

By equating equation (10) at $\theta=90^{\circ}(\vec{v} \perp \hat{r})$ and equation (12), the optimum speed of the magnetic monopole to prevent the collapse of the electron-proton substructure is given by

$$
v=\frac{e}{m_{v}}
$$

to provide enough background vacuum electric field strength to keep the electron afloat from the proton. 
For the charge of the electron $1.6021766 \times 10^{-19}$ Coulomb and the measured magnetic monopole charge $1.463 \times 10^{-35}$ Weber , the speed of the magnetic monopole neutrino turns out to be $1.095 \times 10^{16} \mathrm{~m} / \mathrm{sec}$, which is $3.652 \times 10^{7}$ times the speed of light to be able to stabilize the hydrogen atom and/or all atoms since there is no dependency on $r$ in relation (13).

\section{Reconstruction of the Symmetric Form of Maxwell's Equation}

From this result, we are able to reconstruct the symmetric form of Maxwell's equation by removing the asymmetry between the electricity and magnetism.

$$
\begin{aligned}
& \nabla \cdot \vec{E}=\frac{\rho_{e}}{\varepsilon_{0}} \\
& \nabla \cdot \vec{B}=\mu_{0} \rho_{m} \\
& \nabla \times \vec{E}=-\frac{1}{\varepsilon_{0}} \frac{\partial \vec{B}}{\partial t}-\frac{1}{\varepsilon_{0}} \vec{J}_{m} \\
& \nabla \times \vec{B}=\mu_{0} \vec{J}_{e}+\mu_{0} \frac{\partial \vec{E}}{\partial t}
\end{aligned}
$$

where $\rho_{m}$ is the static magnetic monopole charge density formed by neutrons and $\vec{J}_{m}$ is the magnetic monopole current density from traveling neutrinos. The conventional expression $\nabla \cdot \vec{B}=0$ could pass without noticeable inconsistency because $\rho_{m}$ is extremely weak and individually undetectable unless the collective effect from a massive number of neutrons is subjected to the test. $\vec{J}_{m}$ could also be omitted in the expression for $\nabla \times \vec{E}$ without apparent conflict because there is no "net" observable magnetic current density due to the isotropy of neutrinos flux in free space.

Magnetic current is observable in the form of magnetic flux when the coherent collimated stream of magnetic monopoles passes along the magnet, forming an air gap loop. The actual effect of the electrical current in a typical copper magnet wire according to equation (14) is to rearrange the flow of the random magnetic current that already exists in space into a coherent collimated stream instead of creating the entire magnetic flux from the empty space.

Similar extensions of Maxwell's equation have already been proposed earlier by other researchers [17] who were searching for symmetry between electricity and magnetism. However, they fell short of identifying what the physical quantities $\rho_{m}$ and $\vec{J}_{m}$ represent among the known elementary particles by providing experimental evidence to account for the seemingly unrelated physical phenomena based on their extended form of Maxwell's equations. 


\section{Vacuum Electric Field and Equation of Motion for Electron in Hydrogen Atom}

The vacuum electric field $\vec{E}(\vec{r}, t)$ created by the fast traveling magnetic monopole tachyonic neutrinos can be written as

$$
\vec{E}(\vec{r}, t)=\sum_{i=1}^{N} \frac{1}{4 \pi \varepsilon_{0}} \frac{m_{v} \vec{v}_{i} \times\left(\vec{r}-\vec{r}_{i}(t)\right)}{\left|\left(\vec{r}-\vec{r}_{i}(t)\right)\right|^{3}}
$$

which is an extension of equation (10), where $m_{v}$ is the magnetic monopole charge of the neutrino, $\vec{v}_{i}$ is the velocity, $\vec{r}_{i}(t)$ is the position of the particle at time $t$ and $N$ is the total number of magnetic monopole electron neutrinos in the universe which is estimated to be in the order of $10^{79}$.

This form of background vacuum electric field can provide a rigid medium for electromagnetic wave propagation since the electric field cancels out directionally on the macroscopic scale but not the strength of the field itself in the presence of randomly traveling high-density magnetic monopole neutrinos in the universe. The strongly compressed electric field in vacuum due to large numbers and superluminal speed of magnetic monopole neutrinos accumulates electric energy in space, and it could manifest itself as a fast expanding universe since nature will find ways to reduce its local energy density and separating distance among themselves can certainly fulfill the task.

After all, this vacuum energy must be the enigmatic missing dark energy since the both are performing the same task under the different connotations.

It has also been a puzzle in the theory of electricity and magnetism why measurable physical quantities vacuum permittivity $\varepsilon_{0}$ and permeability $\mu_{0}$ exist in the presumably absolute empty space. Equation (15) shows that the space is filled with fast traveling magnetic monopole neutrinos of charge $m_{v}$, which is directly related to vacuum permeability by $\mu_{0}=n_{0} m_{v}$ (8).

The equation of motion of a free electron in vacuum can be written as

$$
m_{e} \frac{d^{2} \vec{r}}{d t^{2}}+\frac{e}{4 \pi \varepsilon_{0}} \sum_{i=1}^{N} \frac{m_{v} \vec{v}_{i} \times\left(\vec{r}-\vec{r}_{i}(t)\right)}{\left|\left(\vec{r}-\vec{r}_{i}(t)\right)\right|^{3}}=0
$$

, which may define the inertial mass of the electron in the vacuum. The motion of the electron in free space is restricted by the presence of the rigid fluctuating electric field, yet the electron's position and momentum cannot be precisely determined on the microscopic scale. 
The fact that the quantum particle electron's position and momentum in free space are at the whim of magnetic monopole current of neutrinos that have random characteristics in motion indicates that this could be a precursor of the cause of quantum mechanical uncertainty.

The equation of motion of an electron in an isolated hydrogen atom in space can now be written as

$$
m_{e} \frac{d^{2} \vec{r}}{d t^{2}}+\frac{e}{4 \pi \varepsilon_{0}} \sum_{i=1}^{N} \frac{m_{v} \vec{v}_{i} \times\left(\vec{r}-\vec{r}_{i}(t)\right)}{\left|\left(\vec{r}-\vec{r}_{i}(t)\right)\right|^{3}}+\frac{e^{2}}{4 \pi \varepsilon_{0} r^{2}}=0
$$

where $m_{e}$ is the mass of the electron and $e$ the single electronic charge.

Equations (16) and (17) may be reduced into probabilistic statistical forms because there are no means to predict the precise motions of each and every individual tachyonic magnetic monopole particle in the universe other than the physical constraints that they are expected to observe collectively.

In general, fermions do not have a fixed speed of travel, and one may expect tachyonic magnetic monopoles to have a Maxwell-Boltzmann type velocity distribution peaking around $1.095 \times 10^{16} \mathrm{~m} / \mathrm{sec}$ and tapering off at both the speed of infinity and speed of light at the low end. However, since tachyonic neutrinos do not have inertial mass, such as matter particles, nor their motion can be influenced by ambient temperature, their velocity distribution could be radically different and close to a delta function peaking at $1.095 \times 10^{16} \mathrm{~m} / \mathrm{sec}$, in addition to the condition $\sum_{i=1}^{N} m_{v} \vec{v}_{i}=0$.

On the other hand, the arguments against the legitimacy of quantum mechanics, for example, action at far great distance, non-locality, quantum entanglement, hidden variables and incompleteness of quantum mechanics [20], are expected consequences of the theoretical efforts to incorporate the inherent interference from the superluminal magnetic monopoles in the space that is missing in the local probabilistic description of the classical quantum mechanics.

\section{Discussion}

The experimental measurement of the magnetic monopole charge of a neutron makes a starting point to challenge the decades puzzling old dogma of classical quantum mechanics. In fact, in addition to the prediction of the existence of a magnetic monopole by Dirac [6] and many others [7][8], the possibility of the existence of tachyonic particles is already imbedded in Einstein's relativistic mass-energy equation, where the mathematical imaginary "numbered" mass represents particles that travel always faster than the speed of light. When tachyons lose energy, they travel faster as if losing 
"weight" makes them more superluminal according to the special relativistic mass-energy relation,

$$
v^{2}=c^{2}\left(1-\frac{m_{0}{ }^{2} c^{4}}{E^{2}}\right)
$$

The paradox here is that the low-energy neutrinos travel faster and create a stronger electric field around them according to Maxwell's symmetric equation (14). The repulsive magnetic force among individual magnetic monopole neutrinos is negligible, yet the compressed electric field and the energy they create in space are very large due to their enormous speed and numbers. The stable atomic structures and material universe we observe cannot exist without such paradoxical arrangement since the quantum effect is the basis of the existence of the material universe.

Dirac predicted the presence of positron based on his mathematical equation that later has proven to be true. This is an example showing that physical reality that is hidden behind benign looking equations can be more penetrating than rigid interpretation based on traditional belief.

In fact, the mass of a matter particle could be merely a mathematical parameter that determines its physical property depending on the mechanics of its motion. It could also signify the existence of a new class of particles in the universe that do not observe special relativity in the same way as other matter particles. If tachyonic magnetic monopole neutrinos are the ones that provide the property of inertial mass to other elementary particles in the first place, there is no reason for neutrinos to share the same mathematical attributes for their mass as other matter particles.

At the time of this writing, the neutrino is widely considered a spurious elementary particle that exists for no apparent reason other than to satisfy the energy momentum conservation in the nuclear beta decay and in other nuclear interactions. Neutrinos have been observed to change flavors while traveling in space, especially when they present out of the South Pole of the Earth according to a recent experimental report [21]. Since neutrino's magnetic monopole is north kind according to the present report, neutrinos can gain energy by traveling from the Earth's North Pole to the South and change its flavor.

The neutrino is believed to have no effect on quantum mechanical uncertainty. It is not expected to provide a medium for light propagation in empty space nor has any magnetic property according to the current understanding of the universe. The symmetric form of Maxwell's equations (14) with the magnetic monopole current density $\vec{J}_{m}$ of electron neutrinos in place seems to provide the interrelations among these seemingly unrelated and long-held puzzles in physics.

\section{Conclusion}


The measurement detail of the Earth's magnetic monopole charge is presented in relation to the expectation of the magnetic monopole tachyonic neutrino hypothesis in the universe according to the known theoretical conclusion of the charge conservation principle. New symmetric Maxwell's equation is proposed where the symmetry between the electricity and magnetism is fully restored and the magnetic charge density $\rho_{m}$ and magnetic current density $\vec{J}_{m}$ are identified with the measurable physical quantities of the known particles.

The electromagnetic origin of the quantum mechanical uncertainty and the medium for electromagnetic wave propagation are presented by employing the same concept of the magnetic monopole tachyonic neutrinos.

In conclusion, it seems that the overall concept of tachyonic magnetic monopole neutrinos has the theoretical advantage of addressing the long held mechanical mysteries in the universe without adding new contradictions, and it may provide us with an example showing that nature hardly wastes any material or immaterial substance within its boundary to demonstrate its mysterious workings of the universe.

\section{References}

[1] Steyaert, J.J. 1988 The Neutrino as a Tachyonic Non-charged Light Magnetic Monopole? In: Klapdor H.V., Povh B. (eds) Neutrino Physics. Springer, Berlin, Heidelberg

[2] Chodos, Alan; Hauser, Avi I.; Kostelecký, V.A. The neutrino as a tachyon. Physics Letter B 150, 431-435 1985

[3] Recami, E. ed., Tachyons, monopoles and related topics (North-Holland, Amsterdam, 1978) and references therein.

[5] Bilaniuk, O.M.P., V.K. Deshpande and E.C.G. Sudarshan, Am.J. Phys. 30 (1962) 718; O.M.P. Bilaniuk and E.C.G. Sudarshan, Phys. Today 5 (1969) 43.

[6]. Dirac, P. A. M. 1931. Proc. R. Soc. London A 133 : 60

[7]. Polyakov, A. M. 1974. JETP Lett. 20: 194

[8] 't Hooft, G. 1974. Nucl. Phys. B 79 : 276 4. Georgi, H., Glashow, S. L. 1974. Phys. Rev. Lett. $32: 438$

[9] Simpson, J.J. in: Third workshop on grand unification, eds. P.H. Frampton, S.L. Glashow and H. van Dam (Birkh iuser, Basel, 1982)p. 258.

[10] Pati, Jogesh C.; Salam, Abdus (1 June 1974). "Lepton number as the fourth "color"'". Physical Review D. American Physical Society (APS).10(1): 275289.doi:10.1103/physrevd.10.275.ISSN0556-2821.

[11] Polchinski, Joseph. String Theory: Volume I. Cambridge University Press, p. 4. 
[12] Arthur Loureiro, Andrei Cuceu, Filipe B. Abdalla, Bruno Moraes, Lorne Whiteway, Michael McLeod, Sreekumar T. Balan, Ofer Lahav, Aurélien Benoit-Lévy, Marc Manera, Richard P. Rollins, and Henrique S. Xavier Phys. Rev. Lett.123, 0813012019

[13] Talha Ahmed Khan, Kushairy Kadir, Muhammad Alcm, Zeeshan Fchiihid, M. S. Mazliham Geomagnetic field measurement at earth surface: International Conference on Engineering Technology and Technopreneurship (ICE2T) 2017

[14] Glatzmaier, Gary A.; Roberts, Paul H. (1995). "A three-dimensional self-consistent computer simulation of a geomagnetic field reversal".Nature.377(6546): 203209.Bibcode:1995Natur.377..203G. doi:10.1038/377203a0.

[15] "Basic Relationships". Geophysics.ou.edu. Archived from the original on 2010-0709. Retrieved 2009-10-19.

[16] ABUNDANCE OF ELEMENTS IN THE EARTH'S CRUST AND IN THE SEA,CRC Handbook of Chemistry and Physics, 97th edition (2016-2017), p. 14-17

[17] A. I. Arbab, Complex Maxwell's Equations, Chinese Physics B, Volume 22, Number 3 (2013),

I. Bialynicki-Birula and Z. Bialynicka-Birula, Magnetic Monopoles in the Hydrodynamic Formulation of Quantum Mechanics, Physical Review D, Volume 3, Number 10, 15 May 1971 ,

Henning F. Harmuth, Beate Meffert. The modified Maxwell equations (1985) [18] Uggla, Claes (2006)."Spacetime Singularities".Einstein Online.2 (1002). Archived from the original on 2017-01-24. Retrieved 2015-10-20.

[19] Jackiw, R. Dirac's Magnetic Monopoles (Again) https://arxiv.org/pdf/hepth/0212058 2000

[20] Einstein A, Podolsky B, Rosen N; Podolsky; Rosen (1935). "Can QuantumMechanical Description of Physical Reality Be Considered Complete?".Phys.

Rev.47(10): 777-780.Bibcode:1935PhRv...47.777E.doi:10.1103/PhysRev.47.777.

[21] IceCube Collaboration (2013). "Evidence for High-Energy Extraterrestrial Neutrinos at the IceCube Detector". Science. 342(6161): 1242856. arXiv:1311.5238 